\title{
A Matrix Model for Effective of Project Management
}

\author{
Samuel Y. Ruan, Yuan-Ho Chen* \\ Department of Business Administration \& Master's Program in Business Administration \\ Lunghwa University of Science and Technology, Taiwan \\ ${ }^{*}$ Corresponding author's email: yuanhol68 [AT] gmail.com
}

\begin{abstract}
For earned value management (EVM), when an organization attaches nonequal importance to the schedule performance index (SPI) and cost performance index (CPI) of a project, a nonequal-weighted conversion rate between the SPI and CPI is formed. This study employed EVM to integrate SPIs and CPIs to establish a matrix project performance assessment model with a nonequal-weighted conversion rate between the SPI and CPI. This model improves the disadvantages of conventional performance assessment models and enables project managers to simultaneously monitor and control the costs and schedules of their projects. The results provide a practical project performance assessment model for various industries and a basis for developing a project performance assessment information system, thus confirming the practicality and applicability of this study.
\end{abstract}

Keywords--- Earned Value Management (EVM), Schedule Performance Index (SPI), Cost Performance Index (CPI)

\section{INTRODUCTION}

Earned value management (EVM) is a tool and a technique used for monitoring and managing project performance. Christensen (1998) addressed the costs and benefits of using EVM. Anbari (2003), Fleming and Koppelman (2005), and the Project Management Institute have detailed the benchmarking processes in EVM. Lipke et al. (2009) reviewed and confirmed the main concept of EVM. All these aforementioned studies have indicated that the scope, cost, and schedule performance indices of a project can be simultaneously integrated through EVM to effectively control the performance of and deviations from the project schedule and the costs of the project. Pajares and LopezParedes (2011) combined EVM with project risk management to control and monitor project performance.

EVM clarifies the tasks in a project before it is started and integrates the project schedule and cost performance. EVM enables a project manager to simultaneously observe the schedule and cost efficiencies of a project to determine its actual status. Therefore, the project manager can promptly and accurately identify the hidden risks within project schedules and costs, reduce the loss incurred by the risks, and correct the flaws in the project, thereby directing the project to an appropriate direction.

The management performance of a project is critical to its success. Therefore, the performance of an ongoing project must be monitored constantly, and a comprehensive, transparent, and easy-to-understand project performance assessment model must be established.

\subsection{Earned Value Management}

\section{LITERATURE REVIEW}

EVM analysis is an advanced project management technique that evaluates the schedule and cost performance of a project. According to the Project Management Body of Knowledge, EVM is a type of project performance assessment technique.

The basic elements of EVM are the budgeted cost of a work scheduled (BCWS; also known as planned value, PV), budgeted cost of work performed (BCWP; also known as earned value, EV), actual cost of work performed (ACWP; also known as actual cost, $\mathrm{AC}$ ), budget at completion (BAC), estimate at completion (EAC), and estimate to completion (ETC).

Figure 1 illustrates the details of EVM as well as the implications of the aforementioned parameters in project management and their relationships with one another. Table 1 lists the definitions of EVM parameters. 


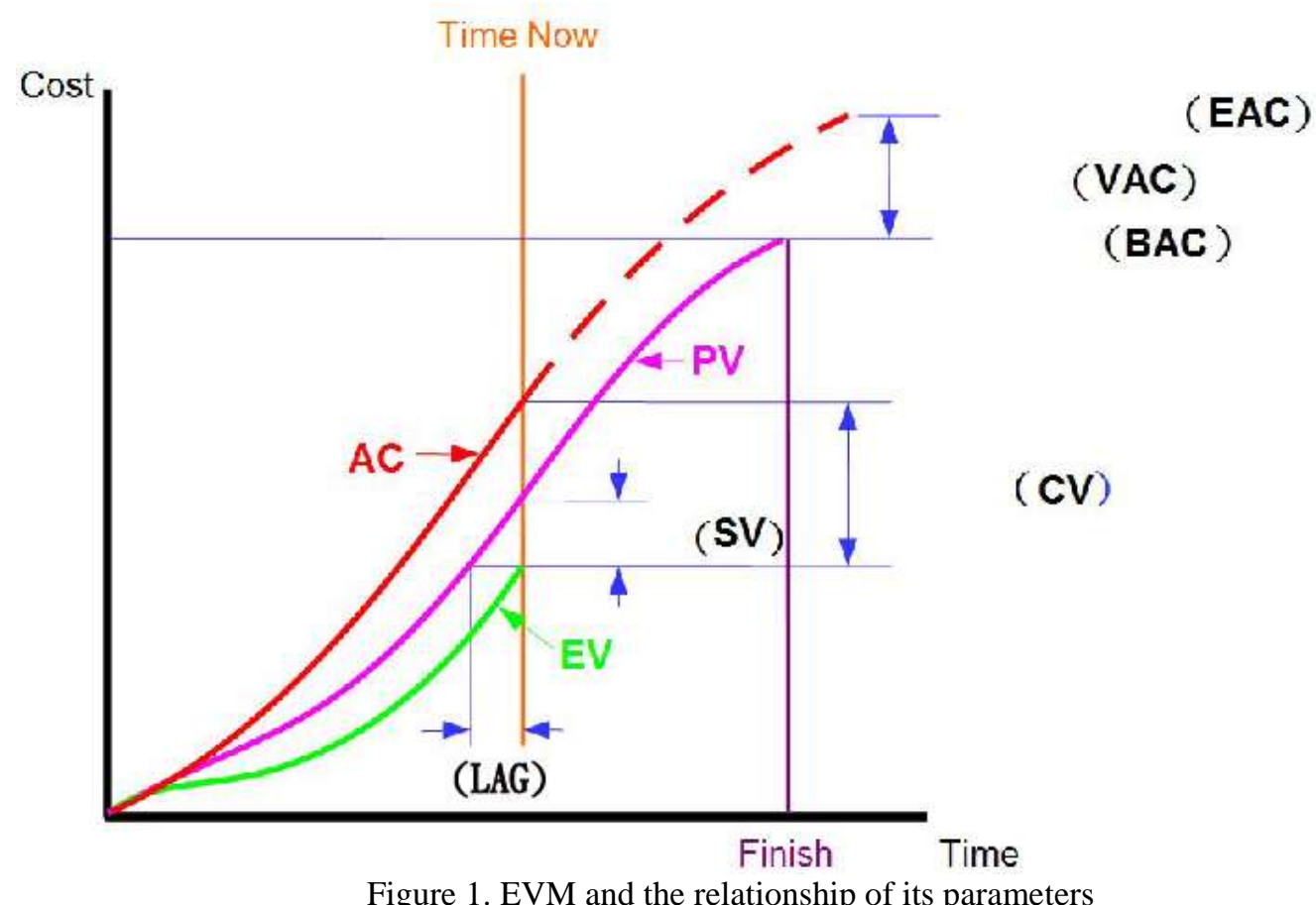

Table 1 Definitions of EVM parameters

\begin{tabular}{|c|c|c|c|}
\hline \multicolumn{2}{|c|}{ Abbreviation } & \multirow{2}{*}{$\begin{array}{l}\text { Full Name } \\
\begin{array}{l}\text { Budgeted Cost for Work } \\
\text { Scheduled }\end{array}\end{array}$} & \multirow{2}{*}{$\begin{array}{l}\text { Definition } \\
\text { Budgeted cost in the project } \\
\text { schedule before the completion } \\
\text { date }\end{array}$} \\
\hline BCWS & $(\mathrm{PV})$ & & \\
\hline BCWP & $(\mathrm{EV})$ & $\begin{array}{l}\text { Budgeted Cost for Work } \\
\text { Performed }\end{array}$ & $\begin{array}{l}\text { Budgeted costs of the } \\
\text { accomplished or partially } \\
\text { completed tasks before the } \\
\text { completion date }\end{array}$ \\
\hline ACWP & $(\mathrm{AC})$ & Actual Cost & $\begin{array}{l}\text { Actual costs of the accomplished } \\
\text { or partially completed tasks before } \\
\text { the completion date }\end{array}$ \\
\hline BAC & & Budget at Completion & $\begin{array}{l}\text { Upper limit of the available } \\
\text { budget }\end{array}$ \\
\hline EAC & & Estimate at completion & $\begin{array}{l}\text { EAC = ACWP }+ \text { Sum of the actual cost and the } \\
\begin{array}{l}\text { BAC }- \text { BCWP) / estimated cost of the unfinished } \\
\text { CPI } \\
\text { tasks on a specific date }\end{array}\end{array}$ \\
\hline ETC & & Estimate to completion & $\begin{array}{l}\text { ETC }=(\text { BAC- Estimated cost of the unfinished } \\
\text { BCWP }) / C P I \quad \text { tasks calculated on a specific date }\end{array}$ \\
\hline SV & & Schedule variance & 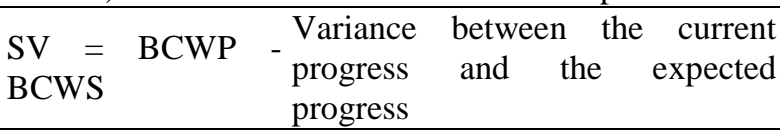 \\
\hline $\mathrm{CV}$ & & Cost variance & $\begin{array}{l}\mathrm{CV}=\mathrm{BCWP} \begin{array}{c}- \text { Variance between the budgeted } \\
\text { cost of the task and its actual cost }\end{array} \\
\mathrm{ACWP}\end{array}$ \\
\hline VAC & & Variance at Completion & $\begin{array}{cc}\text { VAC }=\text { BAC } \begin{array}{l}\text { Estimated cost variance at } \\
\text { completion according to the } \\
\text { performance observed before the } \\
\text { completion date }\end{array}\end{array}$ \\
\hline$\%$ Done & & Percent complete & $\begin{array}{l}\% \text { Done }=\mathrm{BCWP}^{\text {Percentage of task completion }} \\
\text { /BAC }\end{array}$ \\
\hline CPI & & Cost Performance index & $\begin{array}{l}\text { CPI = BCWP } / \text { Ratio of the budgeted cost to the } \\
\text { ACWP }\end{array}$ \\
\hline SPI & & Schedule Performance index & $\begin{array}{llll}\text { SPI = BCWP } & / \text { Ratio of completed tasks } & \text { to } \\
\text { BCWS } & \text { scheduled tasks } & & \end{array}$ \\
\hline
\end{tabular}




\subsection{Research on and Application of Earned Value Management Worldwide}

Numerous studies have been conducted on EVM-based project performance assessment in other countries. For developing a matrix assessment model that simultaneously monitors the progress and cost of a project on a single chart, the present study compiled and reviewed previous studies on EVM. A review is presented as follows:

Naeni et al. (2011) proposed a fuzzy approach for EVM. EVM, a key technique for analyzing and controlling project management performance, enables accurately measuring the progress and performance of a project. Therefore, in accordance with the fuzzy EVM model, the development and advantages of EVM as well as the uncertainty of time and cost estimation were addressed. Because real-life activities involve uncertainties, the findings by Naeni et al. (2011) are specifically applicable for projects with high levels of uncertainty.

Although EVM provides methods for predicting the ultimate costs of projects, the accuracy of these methods cannot be verified when exceptions occur, particularly during the initiation stage of a project. Moreover, EVM cannot be used to predict the duration of each activity scheduled in a project. Lipke et al. (2009) applied a statistical method in EVM and for the SPI to predict the result of a project. To improve the capability of project managers to perform informed decisions, Lipke et al. (2009) formulated an approach to predict the ultimate costs and durations of projects. This approach, along with its evaluation, involved the use of a well-established project management method, a recently developed technique for schedule performance analysis, and a statistical method to improve the accuracy of estimations made using the EVM, earned schedules, and statistical prediction and testing methods. The calculation method was tested using the data of 12 projects. The ultimate costs and durations of the projects calculated using the proposed method provided a reliable reference for determining the tasks, costs, and durations involved in crucial projects.

Bagherpour et al. (2010) designed an EVM-based production environment control mechanism to model the uncertainty pertaining to the durations of activities. This mechanism was designed to solve problems in project management using production control. Bagherpour et al. (2010) regarded job processing time as triangular fuzzy numbers and associated project costs as a fuzzy value dependent on schedule durations. A directly related cost base was estimated according to a hierarchical structure. The results of the research provided an efficient and highly applicable production control mechanism for EVM. The production performance index employed in the production procedures also further predicted the time of product delivery to customers. The control mechanism was verified to be highly applicable after it was employed in solving multiperiod and multiproduct production planning problems.

\section{METHOD}

\subsection{Matrix Project Performance Control Chart}

A project performance control chart is illustrated as a matrix in Figure 4. This control chart surpasses the conventional statistical method (Figure 3) in the following characteristics:

1. Through the respective designation of the $x$-axis and $y$-axis as the CPI and SPI, these two indices can be easily integrated for evaluating the performance and management implications of an ongoing project.

2. The spot at which CPI $=1$ and SPI $=1$ is designated as the central point $(\mathrm{CPI}=1, \mathrm{SPI}=1$ indicates that the current schedule and cost performance of the ongoing project is as satisfactory as the initial plan). The matrix is divided into four quadrants, each of which is defined so that a project team can easily and rapidly assess the management implications of the current schedule performance of the ongoing project.

3. Each quadrant is subdivided into two zones (e.g., B-1 and B-2 in the second quadrant in Figure 4), the differences and management implications of which are then defined. 


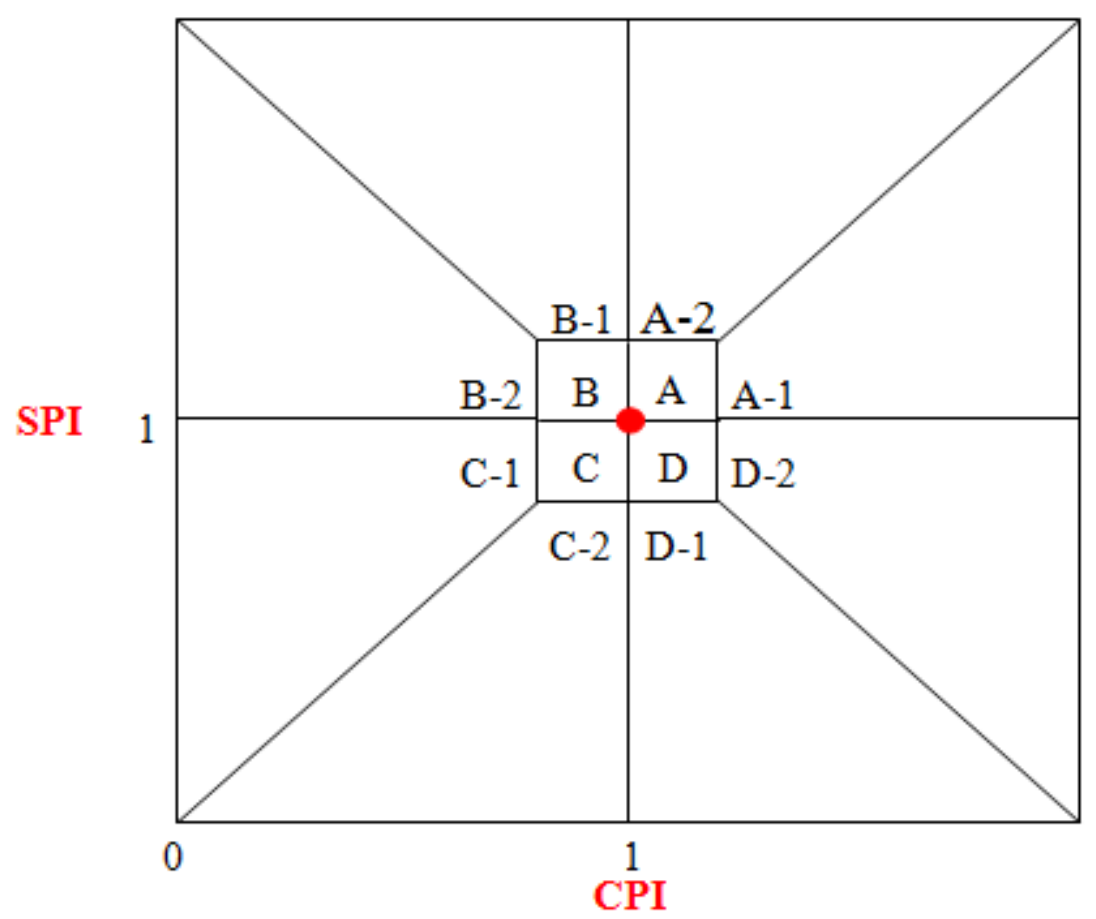

Figure 2. Relationship chart of the SPI and CPI

\subsection{Equal-Weighted and Nonequal-Weighted Conversion Rates Between the Schedule Performance Index and Cost Performance Index}

When an organization or a project manager attaches nonequal importance to the SPI and CPI, the EVM project performance assessment model incorporates a nonequal-weighted conversion rate between SPI and CPI; otherwise, the model employs an equal-weighted conversion rate. This is demonstrated in the following examples:

Example 1. An organization or a project manager attaches equal importance to the SPI and CPI. For example, the management implications for each time point in the following execution process, namely $\mathrm{P}_{\mathrm{ti}}(0.8,1.2) \rightarrow \mathrm{P}_{\mathrm{t} 2}$ $(1.0,1.0) \rightarrow \mathrm{P}_{\mathrm{t} 3}(1.2,0.8)$, are defined as the same by the organization or project manager. Therefore, the project performance assessment model exhibits an equal-weighted conversion rate between SPI and CPI.

Example 2. When the managerial level focuses on the schedule of a project more than its cost, a severe penalty is imposed for a delay in the project schedule, or the project must be finished by a specified date without delays (e.g., Olympic venues), SPI > CPI in terms of importance. Subsequently, $\mathrm{P}_{\mathrm{ti}}(0.8,1.2) \neq \mathrm{P}_{\mathrm{t} 2}(1.0,1.0) \neq \mathrm{P}_{\mathrm{t} 3}$ $(1.2,0.8)$, and the project performance assessment model exhibits a nonequal-weighted conversion rate between the SPI and CPI.

This study focused on the situation with a nonequal-weighted conversion rate between the SPI and CPI. As shown in Figure 3, the $R_{1}$ and $R_{2}$ in the third quadrant should be connected through a nonlinear ellipse equation. For example, when a manager attaches more importance to the schedule performance of a project than to its cost performance (i.e., $\mathrm{SPIr}_{\mathrm{i}}<\mathrm{CPIr}$ ), an elliptic curve results in the quadrant (Figure 3).

The $r_{1}$ and $r_{2}$ risk index control charts are illustrated to demonstrate the extent of the risk acceptance of an enterprise regarding the SPI(SPIr ${ }_{1}$ and $\left.\mathrm{SPIr}_{2}\right)$ and $\mathrm{CPI}(\mathrm{CPIr} 1$ and CPIr2). Each risk index control chart is divided into four zones, namely normal, warning, action, and danger zones. The relative position of the SPI and CPI can be identified on the matrix control chart, in which each position bears a respective management implication. By determining the index positions on the chart, managers can evaluate the current status of an ongoing project and determine whether adjustments and corrections are required. On the basis of the implications of the performance indices, managers can then efficiently control the project and identify tasks that required adjustments or corrective measures.

R1: Performance tolerability. When the actual project performance $(\mathrm{P})$ is lower than $\mathrm{R} 1$, the enterprise should perform corrective measures.

R2: Maximal performance tolerance. When $\mathrm{P}<\mathrm{R} 2$, immediate structural adjustment should be performed for the project, or the project team should determine whether the project must be terminated.

The standard equation for this vertical ellipse is expressed as follows: 
$\frac{x^{2}}{b^{2}}+\frac{y^{2}}{a^{2}}=1 ;(\mathrm{a}>\mathrm{b}>0)$.

Because $(\mathrm{SPI}, \mathrm{CPI})=(1,1)$ was the central point of the chart in this study, the equation after the ellipse was translated is expressed as follows:

$$
\frac{(x-h)^{2}}{b^{2}}+\frac{(y-k)^{2}}{a^{2}}=1 \text {. }
$$

Because $(\mathrm{h}, \mathrm{k})=(1,1)$, the long axis $\mathrm{a}=1-\mathrm{CPI} 1$, and the short axis $\mathrm{b}=1-\mathrm{SPI} 1$,

when $(1-$ SPIr 1$) \leq(1-$ CPIr 1$)$, and CPIr $1<1$, SPIr $1<1$,

the formula should be substituted into the R1 representation as follows:

$$
\frac{(x-1)^{2}}{(1-S P I r 1)^{2}}+\frac{(y-1)^{2}}{(1-C P I r 1)^{2}}=1 \text {. }
$$

when $(1-$ SPIr2 $) \leq(1-$ CPIr2), and CPIr $2<1$, SPIr $2<1$,

the representation of $\mathrm{R} 2$ is as follows:

$$
\frac{(x-1)^{2}}{(1-S P I r 2)^{2}}+\frac{(y-1)^{2}}{(1-C P I r 2)^{2}}=1 \text {. }
$$

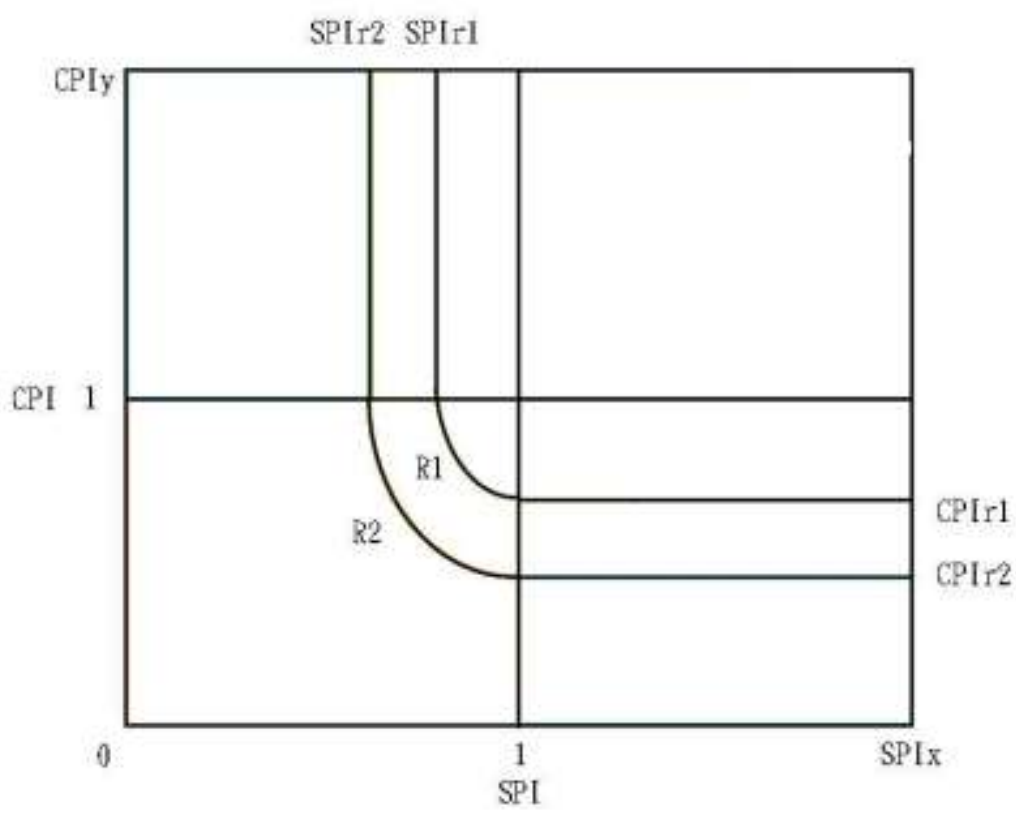

Figure 3. Nonequal-Weighted two-dimensional matrix chart in which the schedule performance of the project is more critical than its cost performance $\left(\mathrm{SPIr}_{\mathrm{i}}<\mathrm{CPIr}\right.$ )

\section{MODEL DEVELOPMENT}

\subsection{Application of the Model Zones in Project Management}

In accordance with the execution statuses of a project, the parameters $\mathrm{SPIr}_{1}, \mathrm{SPIr}_{2}, \mathrm{CPIr}_{1}$, and $\mathrm{CPIr} \mathrm{I}_{2}$ were defined using the two-dimensional matrix chart, and six zones, namely safe, tolerable, correction, change, change or termination, and excessive zones (Figure 3), were established. The parameters were voluntarily determined by the managerial levels of companies or project managers on the basis of project difficulty and company size.

In accordance with the varying statuses of the SPI and CPI, the zones as shown in Figure 4 were derived. Each position corresponding to the zones exhibits a respective management implication. Furthermore, the SPI and CPI in the third quadrant and the zones covered with slash lines are both lower than 1, indicating that a project is behind schedule, and its cost exceeds its budget. Project managers must extensively monitor and control the projects that fall into these zones. The analysis is organized as Figures 3:

1. If the substitution effects of the SPI and CPI are fixed (i.e., the substitution effects of the SPI and CPI cancel 
each other out), then the connecting lines between a (SPIr1, 1) and b (1, CPIr1), between a1 (SPIr2, 1) and b1 (1, CPIr2), are extended as straight or diagonal lines.

2. If the substitution effects of the SPI and CPI are not fixed (i.e., the substitution effect of the SPI to CPI or that of the CPI to SPI increases), then the connecting lines between a (SPIr1, 1) and b (1, CPIr1), between a1 (SPIr2, 1) and b1 (1, CPIr2) are circular or elliptical curves.

3. When the performance of a project falls into the third quadrant (SPI $<1, \mathrm{CPI}<1)$, the project is behind schedule and exhibits cost overrun, and the project should be extensively monitored and controlled. Therefore, three models, namely a circular model, a horizontally elliptical model, and a vertically elliptical model, are employed to assess project performance.

If SPIr1 = CPIr1, and SPIr2 = CPIr2, then the model is circular; both the schedule and cost performance must be strictly controlled to achieve the goal of the project.

If SPIr1 < CPIr1, and SPIr2 < CPIr2, then the model is horizontally elliptical and indicates that the cost performance has been emphasized more than the schedule performance has; the cost performance should be strictly controlled.

If SPIr1 > CPIr1, and SPIr2 > CPIr2, then the model is vertically elliptical and indicates that the schedule performance has been emphasized more than the cost performance has; the schedule performance should be strictly controlled.

On the basis of the sizes and statuses of companies or projects, the SPI-CPI two-dimensional matrix chart was divided into six zones, namely normal, tolerable, correction, change, change or termination, and excessive zones (Figure 6 ), all of which are described as follows:

1. Normal zone: When SPI $\geq 1$ and CPI $\geq 1$ (e.g., $\bullet 1)$, the performance of the project is consistent with the initial plan.

2. Tolerable zone: When $1>$ SPI $>$ SPIr1 and $1>$ CPI $>$ CPIr1 (e.g., $\bullet 2$ ), the performance of the project is less than that of the initial plan but remains within the tolerable range. This project should be continually monitored.

3. Correction zone: When SPIr1 > SPI > SPIr2 and CPIr1 > CPI > CPIr2 (e.g., •3), the performance of the project is less than that of the initial plan and should be adjusted or corrected.

4. Change zone: When SPI $<$ SPIr2 and CPI $\geq 1$ or CPI $<$ CPIr2 and SPI $\geq 1$, the schedule (cost) performance of the project is unsatisfactory, but its cost (schedule) performance is consistent with the initial plan.

5. Change or termination zone: When SPI < SPIr2 and CPI < CPIr2 (e.g., •4), the performance of the project is severely less than that of the initial plan. The project team must thoroughly review this project and determine whether the project should be changed or terminated.

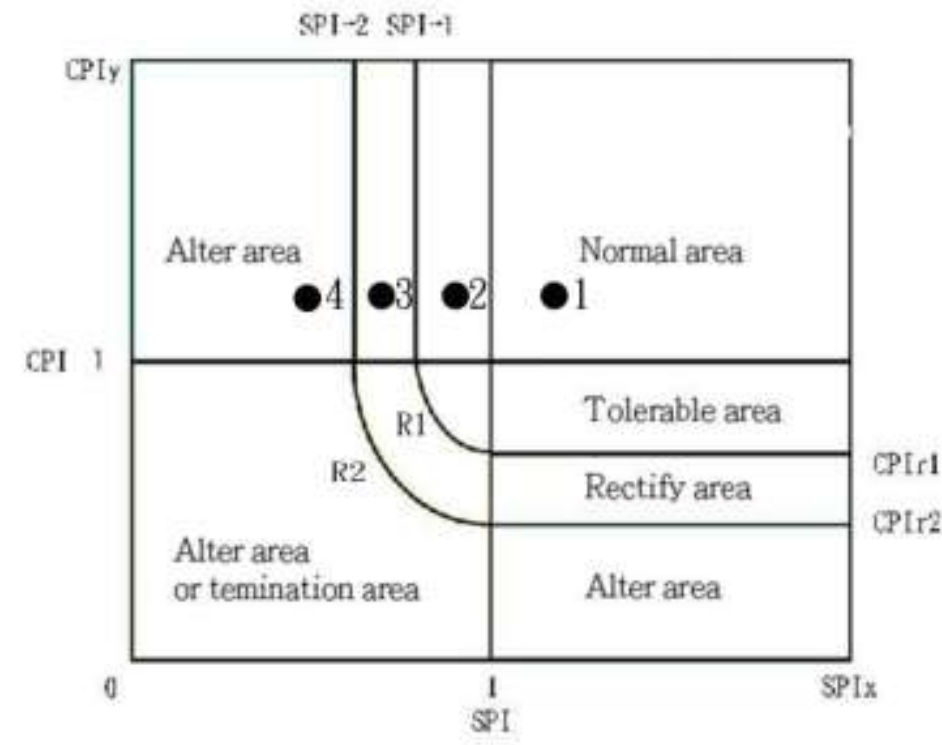

Figure 4. Management implications of the zones in the model 


\section{CONCLUSION}

The assessment model developed in this study was aimed to provide a new method for monitoring and controlling the development of a project. The SPI-CPI two-dimensional matrix relational charts were used to display the development process of a project and thereby enable the project team to prevent and correct flaws in the project.

As revealed by using the assessment model verified in this study, because mobile phones are highly sensitive to their market, their appearances, performance, market acceptance, and competitiveness are the primary factors influencing their market demands. Furthermore, the product lifespans of mobile phones are short, and the difficulty of a new project varies with customer and market demands. Therefore, controlling the schedule and cost of an entire project is challenging and must be operated with extreme flexibility. The project performance assessment model developed in this study is highly applicable for examining project development.

\section{REFERENCES}

1. Anbari, F. T. (2003). Earned value project management method and extensions. Project management journal, 34(4), 12-23.

2. Bagherpour, M, Zareei, A, Noori, S and Heydari, M (2010), Designing a control mechanism using earned value analysis: an application to production environment, International Journal of Advanced Manufacturing Technology, 49 , pp. 419-429.

3. Christensen, D.S. (1998), The costs and benefits of the earned value management process, Acquisition Review Quarterly, Fall, pp. 373-386.

4. Fleming, Q.W. and Koppelman, J.M. (2005), Earned Value Project Management (3rd Edition)Project Management Institute, Newtown Square, PA.

5. Lipke, W., Zwikael, O., Henderson, K. and Anbari, F. (2009), Prediction of project outcome The application of statistical methods to earned value management and earned schedule performance indexes, International Journal of Project Management, 27(4), pp.400-407.

6. Naeni, L.M. , Shadrokh, S. and Salehipour, A. (2011), A fuzzy approach for the earned value management, International Journal of Project Management, 29(6), pp. 764-772 .

7. Pajares, J., \& Lopez-Paredes, A. (2011). An extension of the EVM analysis for project monitoring: The Cost Control Index and the Schedule Control Index. International Journal of Project Management, 29(5), 615-621. 